\title{
Ensemble sufficient dimension folding methods for analyzing matrix-valued data
}

\author{
Yuan Xue $^{\mathrm{a}}$, Xiangrong Yin $^{\mathrm{b}, *}$, Xiaolin Jiang ${ }^{\mathrm{c}}$ \\ ${ }^{a}$ School of Statistics, University of International Business and Economics, Beijing, China \\ ${ }^{b}$ Department of Statistics, University of Kentucky, Lexington, $K Y$, USA \\ ${ }^{c}$ School of Banking and Finance, University of International Business and Economics, Beijing, China
}

\begin{abstract}
The construction of novel sufficient dimension folding methods for analyzing matrix-valued data is considered. For a matrix-valued predictor, traditional dimension reduction methods fail to preserve the matrix structure. However, dimension folding methods can preserve the data structure and improve estimation accuracy. Folded-outer product of gradient (folded-OPG) ensemble estimator and two refined estimators, folded-minimum average variance estimation (folded-MAVE) ensemble and folded-sliced regression (folded-SR) ensemble are proposed to recover central dimension folding subspace (CDFS). Due to ensemble idea, estimation accuracies are improved for finite samples by repeatedly using the data. A modified cross validation method is used to determine the structural dimensions of CDFS. Simulated examples demonstrate the performance of folded ensemble methods by comparing with existing inverse dimension folding methods. The efficacy of folded-MAVE ensemble method is also evaluated by comparing with inverse dimension folding methods for analyzing the Standard \& Poor's 500 stock data set.
\end{abstract}

Keywords: Central dimension folding subspace, central mean dimension folding subspace, folded-MAVE ensemble, folded-OPG ensemble, folded-SR ensemble.

\section{Introduction}

Ding and Cook (2014) investigated the relationship between the monthly Dow Jones industrial average index change rate and 19 daily stock price change rates over the 30 Dow Jones companies from January 2001 to December 2010. The response is a univariate continuous variable, however, the predictor for each observation is a $19 \times 30$ matrix. Traditional sufficient dimension reduction approaches can be applied to such matrix-valued data by vectorizing the data into a vector. As pointed out by Li, Kim and Altman (2010, hereafter LKA) vectorizing a matrix-/array-valued predictor may lose sufficient information, data structure and related interpretation. LKA (2010) introduced a concept of central dimension folding subspace (CDFS) and

\footnotetext{
* Corresponding author

Email address: yinxiangrong@uky.edu (Xiangrong Yin)
} 
proposed three inverse estimation methods, folded-sliced inverse regression (folded-SIR), foldedsliced average variance estimation (folded-SAVE) and folded-directional regression (folded-DR), to reduce the dimensions of a matrix-/array-valued predictor $X$ as much as possible while preserving its intrinsic structure and the regression relation with a univariate response $Y$. If a predictor is vector-valued, sufficient dimension folding reduces to sufficient dimension reduction (Cook, 1994, 1996 and 1998). Treating the predictor $X$ as matrix-/array-valued instead of vectorizing it can preserve the original data structure, important aspects of interpretation, and reduce the number of parameters to enhance estimation accuracy (LKA, 2010). Along the same line, Pfeiffer, Forzani and Bura (2011) proposed an alternative inverse dimension folding method to folded-SIR and named it longitudinal version of sliced inverse regression (LSIR). Ding and Cook (2014) proposed two inverse approaches, dimension folding principal components analysis (DF-PCA) and dimension folding principal fitted components (DF-PFC), to estimate CDFS.

Let $\operatorname{vec}(\cdot)$ be an operation that stacks a matrix into a vector column by column. Inverse dimension folding approaches require certain conditions such as the linearity condition and the constant variance condition on the marginal distribution of $\operatorname{vec}(X)$. However, these conditions are not always satisfied, which can make the estimate not stable. Xue and Yin (2014) considered forward sufficient dimension folding methods for the conditional mean of $Y$ given $X$, which can attain a more accurate estimate when conditional mean is of interest. Xue and Yin (2014) proposed a concept of central mean dimension folding subspace (CMDFS) and its two local estimation methods: folded-OPG and folded-MAVE in analogy to the development of their counterparts outer product of gradient $(\mathrm{OPG})$ and minimum average variance estimation (MAVE) for a vector-valued predictor (Xia et al., 2002). A more general situation is considered by Xue and Yin (2015) which includes CDFS and CMDFS as two special cases. Xue and Yin (2015) performed sufficient dimension folding in reference to a general functional of the conditional distribution of $Y$ given $X$. By applying sufficient dimension folding in reference to a specific functional, the relation between $Y$ and $X$ reflected in that functional is preserved.

In this article, we consider constructing new methods to estimate CDFS based on the ensemble idea introduced by Yin and $\mathrm{Li}$ (2011) for a vector-valued predictor. Yin and Li (2011) considered a general function family $\mathfrak{F}$ of $Y$, and introduced a probability measure on $\mathfrak{F}$. Functions $f_{1}, \ldots, f_{m}$ are randomly sampled from $\mathfrak{F}$ according to that probability. They assembled the central mean subspaces (CMS) of $f_{l}(Y), l=1, \ldots, m$, given a vector-valued predictor to recover the central subspace (CS). The ensemble idea can be applied to estimate CDFS via a set of CMDFS's as well. Folded-MAVE has three appealing advantages in estimating CMDFS: (1) it can exhaustively estimate CMDFS; (2) there is no strong assumption assumed on the distribution of $X$ and (3) the estimation procedure of folded-MAVE can be broken down into iterative quadratic optimizations and each step has an explicit solution. However, folded-MAVE method 
may not recover directions outside CMDFS. Xue and Yin (2014) pointed out that CMDFS is not invariant under one-to-one transformation of the response variable. Based on the ensemble idea, we can repeatedly use data to obtain a better estimate of CDFS, which is achieved by estimating CMDFS for a sufficient large class of transformations of the response variable. We construct folded-OPG ensemble, folded-MAVE ensemble and folded-sliced regression (folded-SR) ensemble methods to estimate CDFS. The first two ensemble methods are similar to those developed by Yin and $\mathrm{Li}$ (2011), and the folded-SR ensemble method is developed in parallel with its counterpart sliced regression (SR; Wang and Xia, 2008) for a vector-valued predictor.

The rest of this article is organized as follows. In Section 2, we study the ensemble theory on characterizing CDFS for a matrix-valued predictor. In Section 3, we introduce algorithms of folded-OPG ensemble, folded-MAVE ensemble and folded-SR ensemble and propose a cross validation criterion to estimate the structural dimensions of CDFS. In Section 4, we comment on the consistency and convergence rate of folded-MAVE ensemble. Simulation studies and a real data analysis are included in Section 5 and Section 6, respectively, followed by a discussion on characterizing CDFS for an array- or higher dimensional valued predictor in Section 7. A short discussion is in Section 8.

\section{Characterizing CDFS}

In this section, we develop sufficient dimension folding methods to estimate CDFS based on the ensemble idea (Yin and Li, 2011) for a matrix-valued predicator. Definitions and theorems in this section are straightforward extensions from Yin and Li (2011). Let the predictor variable $X$ be a $p \times q$ random matrix with its support $\Omega_{X}$ and $Y$ be an $s$-dimensional random vector with its support $\Omega_{Y}$. Let $\mathfrak{F}$ be a family of functions $f: \Omega_{Y} \rightarrow \mathbb{F} \subseteq \mathbb{R}$, which means that a transformation $f$ can project a vector-valued $Y$ to the scalar field $\mathbb{F}$. Let $P_{\mathcal{S}}$ denote the orthogonal projection onto subspace $\mathcal{S}$. Xue and Yin (2014) denoted CMDFS for the conditional mean $E[f(Y) \mid X]$

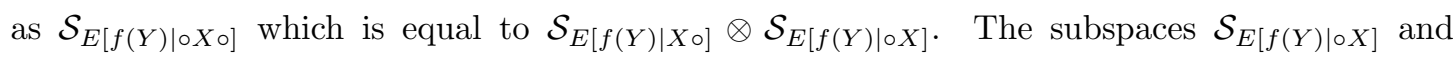
$\mathcal{S}_{E[f(Y) \mid X \circ]}$ are the intersections of all the respective subspaces $\mathcal{S}_{L}$ and $\mathcal{S}_{R}$ of $\mathbb{R}^{p}$ and $\mathbb{R}^{q}$ such that

$$
E[f(Y) \mid X]=E\left[f(Y) \mid P_{\mathcal{S}_{L}} X P_{\mathcal{S}_{R}}\right]
$$

LKA (2010) denoted CDFS of $Y$ versus $X$ as $\mathcal{S}_{Y \mid \circ X \circ}=\mathcal{S}_{Y \mid X \circ} \otimes \mathcal{S}_{Y \mid \circ X}$ where $\mathcal{S}_{Y \mid \circ X}$ and $\mathcal{S}_{Y \mid X \circ}$ are the intersections of all the respective subspaces $\mathcal{S}_{L}$ and $\mathcal{S}_{R}$ of $\mathbb{R}^{p}$ and $\mathbb{R}^{q}$ such that

$$
Y \Perp X \mid P_{\mathcal{S}_{L}} X P_{\mathcal{S}_{R}}
$$

Definition 1. Let $\mathfrak{F}$ be a family of measurable $\mathbb{F}$-valued functions defined on $\Omega_{Y}$. If

$$
\operatorname{span}\left\{\mathcal{S}_{E[f(Y) \mid \circ X \circ]}: f \in \mathfrak{F}\right\}=\mathcal{S}_{Y \mid \circ X \circ}
$$


the family $\mathfrak{F}$ is said to characterize the CDFS.

Our goal is to identify the family $\mathfrak{F}$, so that the dimension folding subspaces for the conditional means $E[f(Y) \mid X]$, when combined for a set of $f(Y)$, can recover the dimension folding subspace for $Y$ versus $X$. Let $F_{Y}$ denote the distribution of $Y$ and $L_{1}\left(F_{Y}\right)$ be the class of functions $f(Y)$ such that $E|f(Y)|<\infty$, with the norm $E|f(Y)|$. Let $L_{2}\left(F_{Y}\right)$ be the class of functions $f(Y)$ with finite variances, with the inner product $<f_{1}, f_{2}>=E\left[f_{1}(Y) f_{2}(Y)\right]$. And let $\mathfrak{B}$ be the family of measurable indicator functions of $Y$, which means $\mathfrak{B}=\left\{I_{\mathcal{B}}: \mathcal{B}\right.$ is a Borel set in $\left.\Omega_{Y}\right\}$. We have the following theorem:

Theorem 1. If $\mathfrak{F}$ is a subset of $L_{2}\left(F_{Y}\right)$ that is dense in $\mathfrak{B}$, then $\mathfrak{F}$ characterizes the CDFS.

Proof. See Appendix A.

There are several function families dense in $L_{2}\left(F_{Y}\right)$ that can be used to characterize CS such as the characteristic functions $\mathfrak{F}=\left\{e^{l t y}: t \in \mathbb{R}\right\}$, where $l=\sqrt{-1}$ (Zhu and Zeng, 2006), the kernel density function $\mathfrak{F}=\operatorname{span}\left\{b^{-1} H[(y-t) / b]: b \in \mathbb{R}^{+}, t \in \mathbb{R}\right\}$, where $H$ is a symmetric probability density function defined on $\mathbb{R}$ (Xia, 2007), and the slices function $\mathfrak{F}=\left\{I_{(-\infty, t)}(y)\right.$ : $t \in \mathbb{R}\}$ (Wang and Xia, 2008). Those families of functions are parametric characterizing families, which means that $\mathfrak{F}$ is of the form $\left\{f_{t}: t \in \Omega_{T}\right\}$ where $\Omega_{T}$ is a subset of a Euclidean space. Those dense families for vector-valued objects can be used to characterize CDFS.

Let $T$ be a random vector defined on $\Omega_{T}$ with distribution $F_{T}$. Suppose that $T_{1}, \ldots, T_{m}$ are random samples from $\Omega_{T}$. Assume that these random elements are defined on a measurable space $(\Omega, \mathcal{A})$, and then $\Omega_{T}$ is interpreted as the range of the mapping $T_{i}: \Omega \rightarrow \Omega_{T}$. We denote a generic member of $\Omega$ by $\omega$. Let $m$ be the number of samplings of function $f$, which we call the ensemble size. The following theorem shows that for a matrix predictor there exists an $m_{0}>0$ when the ensemble size $m \geq m_{0}$, the coverage can be guaranteed.

Theorem 2. Suppose that $\mathfrak{F}$ characterizes the $C D F S$, and $T_{1}, \ldots, T_{m}, \ldots$ are i.i.d. random variables supported on $\Omega_{T}$. For each integer $m, A\left(T_{1}, \ldots, T_{m}\right)$ and $B\left(T_{1}, \ldots, T_{m}\right)$ are orthogonal basis matrices of $\operatorname{span}\left\{\mathcal{S}_{E\left[f_{T_{i}}(Y) \mid \circ X\right]}: i=1, \ldots, m\right\}$ and $\operatorname{span}\left\{\mathcal{S}_{E\left[f_{T_{i}}(Y) \mid X \circ\right]}: i=1, \ldots, m\right\}$, respectively. Then, with probability $1, \mathcal{S}_{Y \mid \circ X} \circ$ can be characterized by a finite number of functions of the response variable in a characterizing family.

\{There is an integer $m_{0}(\omega), \omega \in \Omega$ such that, for all $m \geq m_{0}(\omega)$, $\operatorname{span}\left\{A\left(T_{1}(\omega), \ldots, T_{m}(\omega)\right)\right\}=\operatorname{span}(A)$ and $\left.\operatorname{span}\left\{B\left(T_{1}(\omega), \ldots, T_{m}(\omega)\right)\right\}=\operatorname{span}(B)\right\}$.

Proof. See Appendix B. 
In theory, the "dense" defines classes of sets of functions. Once a dense class is chosen, Theorem 2 states that a finite $m$ should be good enough. We still have two issues: how large of the finite $m$ should be and how we shall select the $m$ functions in practice. We can assign a large ensemble size as long as computational feasible to ensure the stability of the estimation. However, from our limited simulations, when the ensemble size $m=10$, the estimate is already quite stable. Thus, practically, we recommend $m \geq 10$. For the latter, selecting these $m$ functions based on a random sample is a good scheme.

Folded-OPG or folded-MAVE can exhaustively estimate CMDFS, and we apply $m$ functions from a characterizing family to $Y$ to construct the folded-OPG ensemble and folded-MAVE ensemble estimators for CDFS. Choosing the number of slices for sliced regression (SR, Wang and Xia, 2008) method is a challenge. To ease this problem, Cook and Zhang (2014) and Wu and Yin (2015) combined several estimates by SIR (Li, 1991) or SAVE (Cook and Weisberg, 1991) with different numbers of slices together to produce a more accurate estimate of CS. Inspired by their methods, we construct the folded-SR ensemble estimator. That is, applying the slice function to the response variable and combining with different numbers of slices to estimate the CDFS.

\section{Algorithms of sufficient dimension folding ensemble methods}

In this section, we introduce folded-OPG ensemble, folded-MAVE ensemble and folded-SR ensemble estimators. Let $\mathfrak{F}=\left\{f_{t}: t \in \Omega_{T}\right\}$ and $T_{1}, \ldots, T_{m}$ be defined as in Section 2. And let $F_{X}$ be the distribution of $X$. We first assume that $d_{0}$ and $r_{0}$, the structural dimensions of CDFS, are known.

\subsection{Folded-OPG ensemble}

In this section, based on folded-OPG (Xue and Yin, 2014), we consider the folded-OPG ensemble estimator. Its development is based on the fact that the basis matrices of $\mathcal{S}_{E(Y \mid \circ X \circ)}$ can be recovered by estimating the gradient of $E(Y \mid X=x)$, which is achieved by using local linear smoothing (Fan and Gijbels, 1996).

Let $\left\{\left(x_{i}, y_{i}\right), i=1, \ldots, n\right\}$ be i.i.d. observations on $(X, Y)$. And let $K(\cdot)$ be a symmetric probability density function defined on $\mathbb{R}$ - here we use the Gaussian kernel. For any $v \in \mathbb{R}^{p q}$ and $h \in \mathbb{R}^{+}$, we set $K_{h}(v)=h^{-p q} K(\|v\| / h)$, where $h$ is the bandwidth. For folded-OPG ensemble, we minimize the objective function

$$
\sum_{i=1}^{n} w_{i j}(h)\left[f_{T_{k}}\left(y_{i}\right)-c_{j k}-a_{j k}^{\top}\left(x_{i}-x_{j}\right) b_{j k}\right]^{2}
$$


over $c_{j k} \in \mathbb{R}, a_{j k} \in \mathbb{R}^{p}$, and $b_{j k} \in \mathbb{R}^{q}$ for each $j=1, \ldots, n$ and $k=1, \ldots, m$. The kernel weights $w_{i j}(h)$ are set as

$$
w_{i j}(h)=K_{h}\left(\operatorname{vec}\left(x_{i}-x_{j}\right)\right) / \sum_{i=1}^{n} K_{h}\left(\operatorname{vec}\left(x_{i}-x_{j}\right)\right) .
$$

Folded-OPG ensemble involves iterations of quadratic minimization steps and its procedures are summarized as follows.

1. Generate the initial values of $a_{j k} \in \mathbb{R}^{p}$, for $j=1, \ldots, n$ and $k=1, \ldots, m$, from $N(0,1)$.

2. Set $b_{j k}^{\top} b_{j k}=1$. For fixed $a_{j k} \in \mathbb{R}^{p}$, the least squares solution of $(3)$, for each $j=1, \ldots, n$ and $k=1, \ldots, m$, is

$$
\left(\begin{array}{c}
\hat{c}_{j k} \\
\hat{b}_{j k}
\end{array}\right)=\left[\sum_{i=1}^{n} w_{i j}(h) \Delta_{i j}\left(a_{j k}\right) \Delta_{i j}^{\top}\left(a_{j k}\right)\right]^{-1}\left[\sum_{i=1}^{n} w_{i j}(h) \Delta_{i j}\left(a_{j k}\right) f_{T_{k}}\left(y_{i}\right)\right],
$$

where $\Delta_{i j}\left(a_{j k}\right)=\left[1,\left(\operatorname{vec}\left(x_{i}-x_{j}\right)\right)^{\top}\left(I_{q} \otimes a_{j k}\right)\right]^{\top}$.

3. For fixed $c_{j k} \in \mathbb{R}$ and $b_{j k} \in \mathbb{R}^{q}$, the estimate of $a_{j k}$ that minimizes (3), for each $j=1, \ldots, n$ and $k=1, \ldots, m$, is

$$
\hat{a}_{j k}=\left[\sum_{i=1}^{n} w_{i j}(h) \Delta_{i j}\left(b_{j k}\right) \Delta_{i j}^{\top}\left(b_{j k}\right)\right]^{-1}\left[\sum_{i=1}^{n} w_{i j}(h) \Delta_{i j}\left(b_{j k}\right)\left(f_{T_{k}}\left(y_{i}\right)-c_{j k}\right)\right],
$$

where $\Delta_{i j}\left(b_{j k}\right)=\left[\left(\operatorname{vec}\left(x_{i}-x_{j}\right)\right)^{\top}\left(b_{j k} \otimes I_{p}\right)\right]^{\top}$.

4. Let $\hat{A}_{(l)}$ and $\hat{B}_{(l)}$ be the first $d_{0}$ and first $r_{0}$ leading eigenvectors of

$$
\sum_{k=1}^{m} \sum_{j=1}^{n} \hat{a}_{j k} \hat{a}_{j k}^{\top} \text { and } \sum_{k=1}^{m} \sum_{j=1}^{n} \hat{b}_{j k} \hat{b}_{j k}^{\top}
$$

obtained in the $l$ th iteration, respectively. Let $\tau_{(l)}=\hat{B}_{(l)} \otimes \hat{A}_{(l)}, \tau_{(l+1)}=\hat{B}_{(l+1)} \otimes \hat{A}_{(l+1)}$. If $\left\|\tau_{(l+1)} \tau_{(l+1)}^{\top}-\tau_{(l)} \tau_{(l)}^{\top}\right\|$ is smaller than some pre-specified tolerance value, such as $10^{-6}$, stop the iteration and set $\hat{A}=\hat{A}_{(l+1)}, \hat{B}=\hat{B}_{(l+1)}$; otherwise, set $l:=l+1$ and go to Step 2 .

\subsection{Folded-MAVE ensemble}

Note that folded-OPG ensemble has simpler computation but less accuracy compared with folded-MAVE ensemble in this section. The estimated basis matrices $\hat{A}$ and $\hat{B}$ of CDFS obtained by folded-OPG ensemble can serve as the initializers for folded-MAVE ensemble. The population level objective function of folded-MAVE ensemble method can be constructed by applying the folded-MAVE procedure to the transformed response $f_{t}(Y)$ and integrating with respect to the distribution $F_{T}$. It is formulated as:

$$
\int_{\Omega_{T} \times \Omega_{X}} E\left\{\left[f_{t}(Y)-c(x)-a^{\top}(x) A^{\top}(X-x) B^{\top} b^{\top}(x)\right]^{2} K_{h}\left((B \otimes A)^{\top} \operatorname{vec}(X-x)\right)\right\} d F_{X}(x) d F_{T}(t) .
$$


We minimize function (4) over $c(\cdot) \in \mathbb{R}, a(\cdot) \in \mathbb{R}^{d_{0}}, b(\cdot) \in \mathbb{R}^{r_{0}}$, and matrices $A \in \mathbb{R}^{p \times d_{0}}$, $B \in \mathbb{R}^{q \times r_{0}}$.

At the sample level, folded-MAVE ensemble is achieved by minimizing the objective function

$$
\sum_{k=1}^{m} \sum_{j=1}^{n} \sum_{i=1}^{n} \rho_{j} \omega_{i j}(h)\left[f_{T_{k}}\left(y_{i}\right)-c_{j k}-a_{j k}^{\top} A^{\top}\left(x_{i}-x_{j}\right) B b_{j k}\right]^{2}
$$

over $\left\{c_{j k} \in \mathbb{R}: j=1, \ldots, n ; k=1, \ldots, m\right\},\left\{a_{j k} \in \mathbb{R}^{d_{0}}: j=1, \ldots, n ; k=1, \ldots, m\right\},\left\{b_{j k} \in\right.$ $\left.\mathbb{R}^{r_{0}}: j=1, \ldots, n ; k=1, \ldots, m\right\}, A \in \mathbb{R}^{p \times d_{0}}$ and $B \in \mathbb{R}^{q \times r_{0}}$. The trimming functions $\left\{\rho_{j}: j=\right.$ $1, \ldots, n\}$ (Xia et al., 2002; Fan, Yao and Cai, 2003; Xia, 2007; Wang and Xia, 2008; Yin and $\mathrm{Li}, 2011)$ are adopted to exclude unreliable samples with too few observations around. In detail, $\hat{\rho}_{j}=\rho\left(\hat{f}\left(x_{j}\right)\right)$, where $\hat{f}\left(x_{j}\right)=n^{-1} \sum_{i=1}^{n} K_{h}\left((\hat{B} \otimes \hat{A})^{\top} \operatorname{vec}\left(x_{i}-x_{j}\right)\right)$, and $\rho(\cdot)$ is a function, such that $\rho(\omega)>0$ if $\omega>\omega_{0}$, and $\rho(\omega)=0$ if $\omega \leq \omega_{0}$ for some small $\omega_{0}>0$. The kernel weights $w_{i j}(h)$ are set as

$$
w_{i j}(h)=K_{h}\left((\hat{B} \otimes \hat{A})^{\top} \operatorname{vec}\left(x_{i}-x_{j}\right)\right) / \sum_{i=1}^{n} K_{h}\left((\hat{B} \otimes \hat{A})^{\top} \operatorname{vec}\left(x_{i}-x_{j}\right)\right) .
$$

Each time when we have a new estimate $\hat{A}$ or $\hat{B}$, we re-estimate the weights in a lower dimensional subspace (Xia et al., 2002; Fan, Yao and Cai, 2003; Wang and Xia, 2008).

For folded-MAVE ensemble, we adopt the same scheme to decide the bandwidth $h$ as Yin and $\operatorname{Li}(2011)$ did. The initial bandwidth $h_{0}$ is set to be proportional to $n^{-1 /(p q+4)}$. To achieve convergence, at the $k$-th step, let the bandwidth $h_{k}=\max \left\{\zeta h_{k-1}, \hbar\right\}$ and $\hbar=\hbar_{0} \times n^{-1 /\left(d_{0} r_{0}+4\right)}$. The constant $\hbar_{0}$ is selected by the rules suggested by Scott (1992).

Minimization of the objective function (5) can be broken down into iterations between four steps. And each quadratic minimization step has an explicit solution. More specifically, for fixed $A \in \mathbb{R}^{p \times d_{0}}, B \in \mathbb{R}^{q \times r_{0}}$, minimizing (5) over $c_{j k}, a_{j k}$ and $b_{j k}$ for $j=1, \ldots, n$ and $k=1, \ldots, m$ jointly is equivalent to minimizing

$$
\sum_{i=1}^{n} \rho_{j} w_{i j}(h)\left[f_{T_{k}}\left(y_{i}\right)-c_{j k}-a_{j k}^{\top} A^{\top}\left(x_{i}-x_{j}\right) B b_{j k}\right]^{2}
$$

individually, which can be achieved by two iterative steps. For fixed $c_{j k} \in \mathbb{R}, a_{j k} \in \mathbb{R}^{d_{0}}$ and $b_{j k} \in \mathbb{R}^{r_{0}}$, minimizing $[5]$ over $A$ and $B$ can be attained by another two steps. We suggest the following folded-MAVE ensemble procedures.

1. Generate the initial values of $a_{j k} \in \mathbb{R}^{d_{0}}$, for $j=1, \ldots, n$ and $k=1, \ldots, m, A \in \mathbb{R}^{p \times d_{0}}$ and $B \in \mathbb{R}^{q \times r_{0}}$ from $N(0,1)$.

2. Set $b_{j k}^{\top} b_{j k}=1$. For fixed $a_{j k} \in \mathbb{R}^{d_{0}}, A \in \mathbb{R}^{p \times d_{0}}$ and $B \in \mathbb{R}^{q \times r_{0}}$, the least squares solution 
of (6) for each $j=1, \ldots, n$ and $k=1, \ldots, m$, is

$$
\begin{aligned}
\left(\begin{array}{c}
\hat{c}_{j k} \\
\hat{b}_{j k}
\end{array}\right) & =\left[\sum_{i=1}^{n} \rho_{j} w_{i j}(h) \Delta_{i j}\left(a_{j k}, A, B\right) \Delta_{i j}^{\top}\left(a_{j k}, A, B\right)\right]^{-1} \\
& \times\left[\sum_{i=1}^{n} \rho_{j} w_{i j}(h) \Delta_{i j}\left(a_{j k}, A, B\right) f_{T_{k}}\left(y_{i}\right)\right]
\end{aligned}
$$

where $\Delta_{i j}\left(a_{j k}, A, B\right)=\left[1,\left(\operatorname{vec}\left(x_{i}-x_{j}\right)\right)^{\top}(B \otimes A)\left(I_{r_{0}} \otimes a_{j k}\right)\right]^{\top}$.

3. For fixed $c_{j k} \in \mathbb{R}, b_{j k} \in \mathbb{R}^{r_{0}}, A \in \mathbb{R}^{p \times d_{0}}, B \in \mathbb{R}^{q \times r_{0}}$, the minimizer of (6), for each $j=1, \ldots, n$ and $k=1, \ldots, m$, is

$$
\begin{aligned}
\hat{a}_{j k} & =\left[\sum_{i=1}^{n} \rho_{j} w_{i j}(h) \Delta_{i j}\left(b_{j k}, A, B\right) \Delta_{i j}^{\top}\left(b_{j k}, A, B\right)\right]^{-1} \\
& \times\left[\sum_{i=1}^{n} \rho_{j} w_{i j}(h) \Delta_{i j}\left(b_{j k}, A, B\right)\left(f_{T_{k}}\left(y_{i}\right)-c_{j k}\right)\right],
\end{aligned}
$$

where $\Delta_{i j}\left(b_{j k}, A, B\right)=\left[\left(\operatorname{vec}\left(x_{i}-x_{j}\right)\right)^{\top}(B \otimes A)\left(b_{j k} \otimes I_{d_{0}}\right)\right]^{\top}$.

4. For fixed $c_{j k} \in \mathbb{R}, a_{j k} \in \mathbb{R}^{d_{0}}, b_{j k} \in \mathbb{R}^{r_{0}}, j=1, \ldots, n, k=1, \ldots, m$, and $A \in \mathbb{R}^{p \times d_{0}}$, the least squares solution of $[5]$ is

$$
\begin{aligned}
\operatorname{vec}(\hat{B}) & =\left[\sum \rho_{j} w_{i j}(h) \Delta_{i j}\left(a_{j k}, b_{j k}, A\right) \Delta_{i j}^{\top}\left(a_{j k}, b_{j k}, A\right)\right]^{-1} \\
& \times\left[\sum \rho_{j} w_{i j}(h) \Delta_{i j}\left(a_{j k}, b_{j k}, A\right)\left(f_{T_{k}}\left(y_{i}\right)-c_{j k}\right)\right],
\end{aligned}
$$

where $\Delta_{i j}\left(a_{j k}, b_{j k}, A\right)=\left[I_{r_{0}} \otimes\left(\left(x_{i}-x_{j}\right)^{\top} A\right)\right]\left(b_{j k} \otimes a_{j k}\right)$ and the summation is over

$$
(i, j, k) \in\{1, \ldots, n\} \times\{1, \ldots, n\} \times\{1, \ldots, m\} .
$$

Normalize $\hat{B}$ so that $\hat{B}^{\top} \hat{B}=I_{r_{0}}$.

5. For fixed $c_{j k} \in \mathbb{R}, a_{j k} \in \mathbb{R}^{d_{0}}, b_{j k} \in \mathbb{R}^{r_{0}}, j=1, \ldots, n, k=1, \ldots, m$, and $B \in \mathbb{R}^{q \times r_{0}}$, the $\hat{A}$ that minimizes (5) is

$$
\begin{aligned}
\operatorname{vec}\left(\hat{A}^{\top}\right) & =\left[\sum \rho_{j} w_{i j}(h) \Delta_{i j}\left(a_{j k}, b_{j k}, B\right) \Delta_{i j}^{\top}\left(a_{j k}, b_{j k}, B\right)\right]^{-1} \\
& \times\left[\sum \rho_{j} w_{i j}(h) \Delta_{i j}\left(a_{j k}, b_{j k}, B\right)\left(f_{T_{k}}\left(y_{i}\right)-c_{j k}\right)\right],
\end{aligned}
$$

where $\Delta_{i j}\left(a_{j k}, b_{j k}, B\right)=\left[\left(\left(x_{i}-x_{j}\right) B\right) \otimes I_{d_{0}}\right]\left(b_{j k} \otimes a_{j k}\right)$ and the summation is over

$$
(i, j, k) \in\{1, \ldots, n\} \times\{1, \ldots, n\} \times\{1, \ldots, m\} .
$$

Normalize $\hat{A}$ so that $\hat{A}^{\top} \hat{A}=I_{d_{0}}$.

6. Check convergence. Let $A_{(l)}, B_{(l)}$ be the estimates of $A$ and $B$ obtained in the $l$ th iteration, respectively. Let $\tau_{(l)}=B_{(l)} \otimes A_{(l)}, \tau_{(l+1)}=B_{(l+1)} \otimes A_{(l+1)}$. If $\left\|\tau_{(l+1)} \tau_{(l+1)}^{\top}-\tau_{(l)} \tau_{(l)}^{\top}\right\|$ is smaller than some pre-specified tolerance value, such as $10^{-6}$, stop the iteration and set $A=A_{(l+1)}, B=B_{(l+1)} ;$ Otherwise, set $l=: l+1$ and go to Step 2 . 


\subsection{Folded-SR ensemble}

In SR method, Wang and Xia (2008) pre-selected grid points $-\infty=s_{0}<s_{1}, \ldots,<s_{H}=+\infty$ to slice the response variable $Y$, which defines a new response variable $z_{t}$ such that $z_{t}=I\left(s_{(t-1)}<\right.$ $Y<s_{t}$ ), where $I(\cdot)$ is the indicator function and $t=1, \ldots, H$. Through estimating the CMS of $z_{t}$, Wang and Xia (2008) achieved the goal to estimate the CS of $Y$ given a vector-valued predictor. However, for sliced methods, choosing a proper number of slices is a challenge.

Based on the ensemble idea (Yin and Li, 2011; Wu and Yin, 2015) and the fused estimators (Cook and Zhang, 2014), we consider a sequence of the number of slices $H=\left\{H_{1}, \ldots, H_{k}\right\}, k=$ $1, \ldots, m$. Each value of the subscript $k$ corresponds to a selection of the number of slices from $\left\{H_{1}, \ldots, H_{m}\right\}$. For each selection of $H_{k}$ and $t=1, \ldots, H_{k}$, we define $z_{(k, t)}=I\left(s_{(k, t-1)}<\right.$ $\left.Y<s_{(k, t)}\right)$, where $-\infty=s_{(k, 0)}<s_{(k, 1)}, \ldots,<s_{\left(k, H_{k}\right)}=+\infty$. The unison of the CMDFSes estimated based on $\left[z_{(k, 1)}, \ldots, z_{\left(k, H_{k}\right)}\right]$ for $k=1, \ldots, m$, covers the CDFS of $Y \mid X$. The sample level objective function is the summation of

$$
\sum_{t=1}^{H_{k}} \sum_{j=1}^{n} \sum_{i=1}^{n} \rho_{j} \omega_{i j}(h)\left[z_{(k, t)}\left(y_{i}\right)-c_{j k}-a_{j k}^{\top} A^{\top}\left(x_{i}-x_{j}\right) B b_{j k}\right]^{2},
$$

over $k=1, \ldots, m$. The objective function is minimized over $c_{j k} \in \mathbb{R}, a_{j k} \in \mathbb{R}^{d_{0}}, b_{j k} \in \mathbb{R}^{r_{0}}$, $A \in \mathbb{R}^{p \times d_{0}}$ and $B \in \mathbb{R}^{q \times r_{0}}$, for $j=1, \ldots, n$ and $k=1, \ldots, m$. Similar iterative procedures as the previous folded-MAVE ensemble can be applied to estimate $A$ and $B$, the basis matrices of $S_{Y \mid \circ X}$ and $S_{Y \mid X \circ}$, respectively.

\subsection{Estimation of CDFS dimensions}

Previously, we assumed that $d_{0}$ and $r_{0}$, the structural dimensions of CDFS, are known. However, these dimensions may need to be estimated in practice. We modify the cross validation method proposed by Yin and Li (2011) to estimate $d_{0}$ and $r_{0}$. Let $\hat{A}$ and $\hat{B}$ be the estimated basis matrices of $\mathcal{S}_{Y \mid \circ X}$ and $\mathcal{S}_{Y \mid X \circ}$ for fixed working dimensions $d$ and $r$, respectively. We assume $d_{0} \neq 0$ and $r_{0} \neq 0$. The leave-one-out fitted value of $f_{T_{k}}\left(y_{i}\right)$, for $j=1, \ldots, n, k=1, \ldots, m$ is

$$
\hat{f}_{T_{k}}\left(y_{i}\right)=\sum_{i \neq j} K_{h}\left[(\hat{B} \otimes \hat{A})^{\top} \operatorname{vec}\left(x_{i}-x_{j}\right)\right] f_{T_{k}}\left(y_{i}\right) / \sum_{i \neq j} K_{h}\left[(\hat{B} \otimes \hat{A})^{\top} \operatorname{vec}\left(x_{i}-x_{j}\right)\right] .
$$

Then the corresponding cross validation value is

$$
C V(d, r)=\frac{1}{m n} \sum_{k=1}^{m} \sum_{j=1}^{n}\left[f_{T_{k}}\left(y_{i}\right)-\hat{f}_{T_{k}}\left(y_{i}\right)\right]^{2} .
$$

And the dimensions $d_{0}$ and $r_{0}$ are estimated by

$$
\left(\hat{d}_{0}, \hat{r}_{0}\right)=\arg \min \{C V(d, r): d=1, \ldots, p ; r=1, \ldots, q\} .
$$




\section{Convergence rate and consistency}

In this section, we investigate the convergence rate of folded-MAVE ensemble estimator assuming $d_{0}$ and $r_{0}$ are known and consistency of the cross validation estimator of $d_{0}$ and $r_{0}$. Although here we only consider folded-MAVE ensemble, the development can be extended to folded-SR ensemble easily. The results are similar to those made in Xia (2007), Wang and Xia (2008), and especially Yin and Li (2011). Following Yin and Li (2011), we consider the asymptotic analysis in two steps. In the first step (Theorem 3), we establish the convergence rate for a fixed set of functions $\left\{f_{t_{1}}, \ldots, f_{t_{m}}\right\}$ in $\mathfrak{F}$. In the second step (Theorem 4), we investigate the asymptotic behavior when $m \rightarrow \infty$.

For any finite set $\left\{t_{1}, \ldots, t_{m}\right\} \subseteq \Omega_{T}$, let $\hat{A}\left(t_{1: m}\right)=\hat{A}\left(t_{1}, \ldots, t_{m}\right)$ and $\hat{B}\left(t_{1: m}\right)=\hat{B}\left(t_{1}, \ldots, t_{m}\right)$ be the folded-MAVE ensemble estimators. And let $A\left(t_{1: m}\right)=A\left(t_{1}, \ldots, t_{m}\right)$ and $B\left(t_{1: m}\right)=$ $B\left(t_{1}, \ldots, t_{m}\right)$ be the basis matrices of $\operatorname{span}\left\{\mathcal{S}_{E\left[f_{t_{i}}(Y) \mid \circ X \circ\right]}: i=1, \ldots, m\right\}$. Without loss of generality, we assume that these matrices are semiorthogonal.

The following theorem gives the convergence rate of folded-MAVE ensemble estimator for a fixed set of functions in $\mathfrak{F}$ and fixed $d_{0}$ and $r_{0}$.

Theorem 3. Suppose conditions (C1), (C2), (C4) and (C5) in Yin and Li (2011) are satisfied for vec(.), and (C3) in Yin and Li (2011) holds for $\left\{t_{1}, \ldots, t_{m}\right\} \subseteq \Omega_{T}$ as well. Then, as $n \rightarrow \infty$,

$$
\begin{aligned}
\lim _{n \rightarrow \infty} & \left\|\left(\hat{B}\left(t_{1: m}\right) \otimes \hat{A}\left(t_{1: m}\right)\right)\left(\hat{B}\left(t_{1: m}\right) \otimes \hat{A}\left(t_{1: m}\right)\right)^{\top}-\left(B\left(t_{1: m}\right) \otimes A\left(t_{1: m}\right)\right)\left(B\left(t_{1: m}\right) \otimes A\left(t_{1: m}\right)\right)^{\top}\right\| \\
& =O_{P}\left[\hbar^{4}+\log n /\left(n \hbar^{d_{0} r_{0}}\right)+n^{-1 / 2}\right] .
\end{aligned}
$$

Ultimately, we are interested in $\mathcal{S}_{Y \mid \circ X \circ}$ instead of $\operatorname{span}\left\{\mathcal{S}_{E\left[f_{t_{i}}(Y) \mid \circ X \circ\right]}: i=1, \ldots, m\right\}$. Let $T_{1}, \ldots, T_{m}$ be an independent sample from $\Omega_{T}$ and they are independent of $\left\{x_{i}, y_{i}\right\}, i=$ $1, \ldots, n$. Let $A$ and $B$ denote the semiorthogonal basis matrices of $\mathcal{S}_{Y \mid \circ X \circ}$, and bandwidth $\hbar \propto n^{-1 /\left(d_{0} r_{0}+4\right)}$. The next theorem shows that, under the conditions no stronger than Theorem 3 , folded-MAVE ensemble recovers the CDFS at the same rate as rMAVE does for a vector-valued predictor.

Theorem 4. Suppose that conditions (C1)-(C5) in Yin and Li (2011) hold for vec(.). Then, for any $\epsilon>0$,

$$
\lim _{m \rightarrow \infty} \lim _{n \rightarrow \infty} P\left(\frac{\left(\hat{B}\left(T_{1: m}\right) \otimes \hat{A}\left(T_{1: m}\right)\right)\left(\hat{B}\left(T_{1: m}\right) \otimes \hat{A}\left(T_{1: m}\right)\right)^{\top}-(B \otimes A)(B \otimes A)^{\top}}{\hbar^{4}+\log n /\left(n \hbar^{d_{0} r_{0}}\right)+n^{-1 / 2}}>\epsilon\right)=0 .
$$

The proofs of Theorem 3 and Theorem 4 can follow Yin and Li (2011) except taking $\hat{B}\left(T_{1: m}\right) \otimes$ $\hat{A}\left(T_{1: m}\right)$ instead of $\hat{B}\left(T_{1}, \ldots, T_{m}\right)$. We omit the proof for brevity. As pointed out, if $d_{0} r_{0}<3$ then $\sqrt{n}$-consistency can be achieved by taking $\hbar \propto n^{-1 /\left(d_{0} r_{0}+4\right)}$. 
Next, we investigate the convergence to the structural dimensions $d_{0}$ and $r_{0}$ as $m \rightarrow \infty$. Let $\hat{d}\left(T_{1: m}\right)=\hat{d}\left(T_{1}, \ldots, T_{m}\right)$ and $\hat{r}\left(T_{1: m}\right)=\hat{r}\left(T_{1}, \ldots, T_{m}\right)$ be the cross validation estimators of $d\left(T_{1: m}\right)=d\left(T_{1}, \ldots, T_{m}\right)$ and $r\left(T_{1: m}\right)=r\left(T_{1}, \ldots, T_{m}\right)$ which are the dimensions for $A\left(T_{1}, \ldots, T_{m}\right)$ and $B\left(T_{1}, \ldots, T_{m}\right)$, respectively.

Theorem 5. Under the assumptions in Theorem 4, we have

$$
\lim _{m \rightarrow \infty} \lim _{n \rightarrow \infty} P\left(\left[\hat{d}\left(T_{1: m}\right), \hat{r}\left(T_{1: m}\right)\right]=\left(d_{0}, r_{0}\right)\right)=1 .
$$

Proof. See Appendix C.

\section{Numerical studies}

In this section, we demonstrate the performance of folded-OPG ensemble, folded-MAVE ensemble and folded-SR ensemble methods by comparing with folded-SIR, folded-SAVE, folded$\mathrm{DR}$ and isotropic DF-PFC on simulated examples. The characteristic function is adopted as the choice of $\mathfrak{F}$ for folded-OPG ensemble and folded-MAVE ensemble. The ensemble size $m$ is set to be 10, unless otherwise stated. The numbers of slices for folded-SR ensemble are set as $H=(5,10,15,20)$. Simulations are carried out by Matlab and $\mathrm{C}++$ language. We assume that $X$ is a random matrix in $\mathbb{R}^{p \times q}$ and $\operatorname{vec}(X) \sim N_{p q}(\mathbf{0}, \Sigma)$, where $\mathbf{0}$ is a $p q \times 1$ vector and $\Sigma$ is a $p q \times p q$ positive definite matrix with the $\left(j_{1}, j_{2}\right)$ th entry $0.5^{\left|j_{1}-j_{2}\right|}$. We write $X_{i j}$ as the random predictor in the $i j$ th position of $X$. The random error $\epsilon$ is a standard normal random variable and independent of $X$. We set $e_{i}$ as a generic vector whose $i$ th element equals 1 and otherwise 0 . We set the size of $X$ as $p=q=3, p=q=5$ and $p=q=7$, unless otherwise stated.

Our methods inherit the advantages and disadvantages of OPG and MAVE approaches, in that they perform the best for moderate sample size and moderate dimensions of $X$. We simulate Example 1 under $p=q=10$ (there are 100 random variables in one matrix predictor, not reported). Our approaches still shows their advantages. However, our proposed methods are local smooth approaches, and as such, if the size of $X$ is too big, then the proposed methods may have trouble due to their computational speed and handling problems of the software.

Without loss of generality, we assume the matrices $A$ and $B$ and their corresponding estimates $\hat{A}$ and $\hat{B}$ to be semiorthogonal. To measure the accuracy of different estimators, we use the matrix norm operator $\Delta(B \otimes A, \hat{B} \otimes \hat{A})=\left\|(B \otimes A)(B \otimes A)^{\top}-(\hat{B} \otimes \hat{A})(\hat{B} \otimes \hat{A})^{\top}\right\|$ proposed by Li, Zha and Chiaromonte (2005). For the matrix norm, we use the Frobenius norm. A value of 0 means that the two subspaces are the same. Thus the smaller the value is, the closer the two subspaces are. We also can use the benchmark distance (Li, Wen and Zhu, 2008) to evaluate the discrepancy between two independent spaces. Let $P_{\alpha_{1}}$ and $P_{\alpha_{2}}$ be the projections onto the column space of 
$\alpha_{1} \in \mathbb{R}^{s \times t}$ and $\alpha_{2} \in \mathbb{R}^{s \times t}$, respectively. Generating i.i.d. $\alpha_{1}$ and $\alpha_{2}$, the benchmark distance is defined as $E\left(\left\|P_{\alpha_{1}}-P_{\alpha_{2}}\right\|\right)$, and it is determined only by the value of $s$ and $t$. We estimate the benchmark distance by running 10,000 simulations, which set a benchmark distribution. We can calculate a "p-value" as the proportion of distances in the distribution whose values are smaller than the value of the matrix norm between the estimated and known subspaces. If the "p-values" is less than 0.05 , we reject the null hypothesis so that the two subspaces are not random, which means the estimate is good, otherwise, the estimate is bad.

We generate 100 replicates data for each example and compute the average and standard error of the Frobenius norm. The accuracy of estimating CDFS is reported as mean and standard error in parenthesis. In addition, we report the percentage of correctly estimating the dimensions $d_{0}$ and $r_{0}$ by the cross validation criterion based on folded-MAVE ensemble method. We use f-OPG-E, f-MAVE-E and f-SR-E to denote the three ensemble folded methods for short in the reported tables. First, to see how $m$ may affect the result, we simulate the following Examples 1 and 2 at different ensemble sizes. In Figures 1 and 2, we plot the averages of $\Delta(B \otimes A, \hat{B} \otimes \hat{A})$ over the 100 simulated samples versus ensemble sizes $m$ at 4, 10, 16, 20 and 40 for Examples 1 and 2, respectively. The size of $X$ is set to be $5 \times 5$ and the sample size is 400 . For Example 1 , since CMDFS is equal to CDFS, the ensemble size has not much effect on the estimation error. Thus the average error is stable for all the choice of $m$. For Example 2, based on the plot the average error is quite stable as $m$ varies among 10,16, 20, and 40 while 10 is the elbow point. Assigning a large ensemble size can ensure the stability but increasing computational cost. The ensemble size $m=10$ seems work well in our limited simulations.

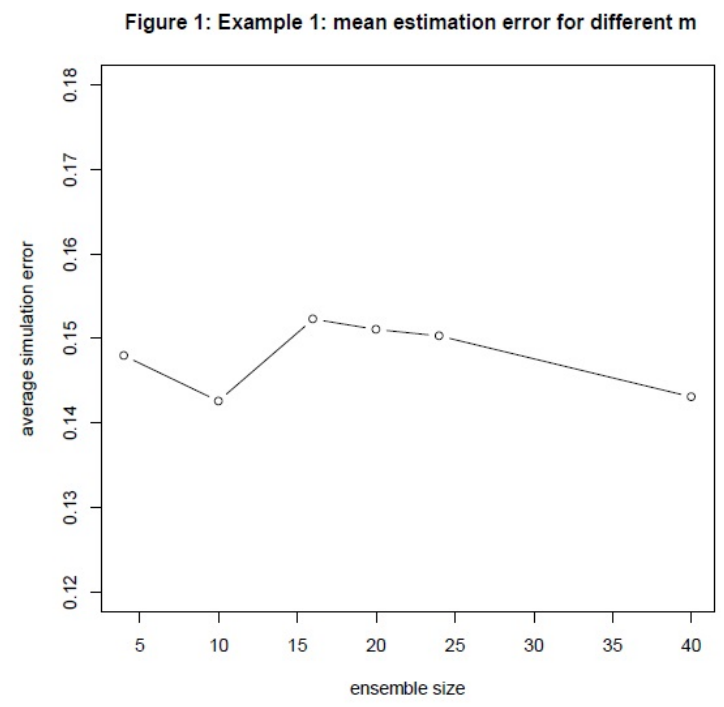




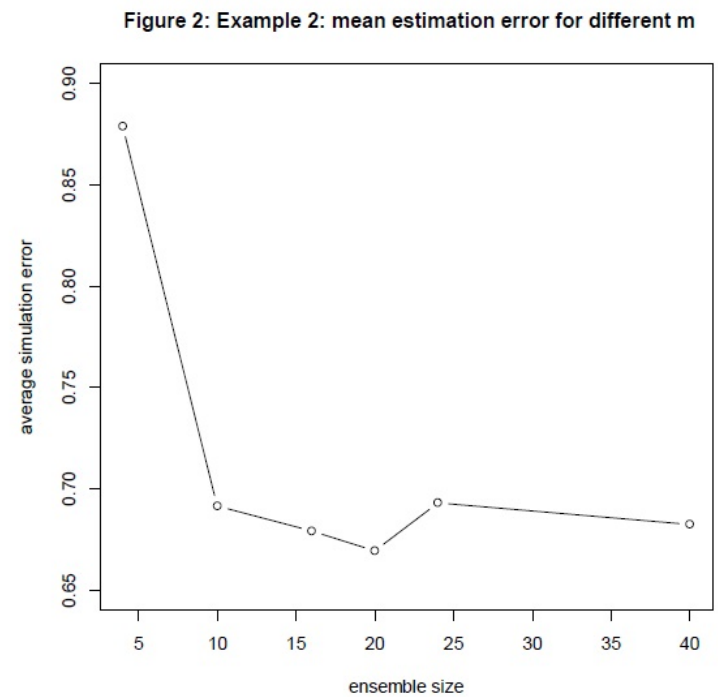

Example 1: $Y=X_{11} \times\left(X_{21}+1\right)+0.2 \epsilon$.

The dimensions of CDFS for this example are $d_{0}=2$ and $r_{0}=1$, and the basis matrices are $A=\left(e_{1}, e_{2}\right) \in \mathbb{R}^{p \times 2}$ and $B=e_{1} \in \mathbb{R}^{q}$. The benchmark distances for $p=q=3, p=q=5$ and $p=q=7$ are 1.7430, 1.9148 and 1.9575 and their corresponding $5 \%$ quantile values are 1.2083 , 1.6889 and 1.8383, respectively. From Table 1, based on benchmark distance it seems that folded-SAVE estimates a random subspace, and other methods correctly estimate the subspace. Table 1 shows that the estimation accuracies of folded-MAVE ensemble and folded-SR ensemble are close to each other and both overtake other methods. Folded-OPG ensemble method is less accurate than the other two ensemble folded methods, but it provides comparable results to folded-SIR and folded-DR.

Example 2: $\quad Y=X_{11} /\left(0.5+\left(X_{12}+1.5\right)^{2}\right)+X_{22} \times \epsilon$

This example is constructed based on Example 3 of Wang and Xia (2008). For this example, the dimensions of CDFS are $d_{0}=r_{0}=2$. The benchmark distances for $p=q=3, p=q=5$ and $p=q=7$ are $2.0854,2.5904$ and 2.7083 , and corresponding $5 \%$ quantile values are $1.4566,2.3104$ and 2.5372, respectively. The basis matrices are $A=\left(e_{1}, e_{2}\right) \in \mathbb{R}^{p \times 2}$ and $B=\left(e_{1}, e_{2}\right) \in \mathbb{R}^{q \times 2}$. Folded-MAVE can only estimate the CMDFS whose two basis matrices are $A=e_{1} \in \mathbb{R}^{p}$ and $B=\left(e_{1}, e_{2}\right) \in \mathbb{R}^{q \times 2}$. The dimensions of CMDFS are $d_{0}=1, r_{0}=2$. Simulation results in Table 2 indicate that folded-MAVE ensemble is consistently the best and folded-SR ensemble is the second. Folded-OPG ensemble provides more accurate estimates than the three inverse folded methods in LKA (2010) and the DF-PFC method in Ding and Cook (2014). Also, based on benchmark distance, folded-SIR, folded-SAVE and DF-PFC may estimate a random subspace.

Example 3: $Y=\cos \left(2 X_{11}\right)+\cos \left(X_{21}\right)-\cos \left(X_{22}\right)+0.2 \epsilon$ 
Table 1: Example 1. accuracy of estimates

\begin{tabular}{|c|c|c|c|c|c|c|c|c|}
\hline Size & $\mathrm{n}$ & f-OPG-E & f-MAVE-E & f-SR-E & folded-SIR & folded-SAVE & folded-DR & DF-PFC \\
\hline \multirow{6}{*}{$3 \times 3$} & \multirow{2}{*}{200} & 0.3314 & 0.1118 & 0.1442 & 0.3376 & 1.4133 & 0.3197 & 0.7380 \\
\hline & & $(0.1333)$ & $(0.0366)$ & $(0.0620)$ & $(0.1575)$ & $(0.4130)$ & $(0.1360)$ & $(0.1791)$ \\
\hline & \multirow{2}{*}{400} & 0.2431 & 0.0802 & 0.0965 & 0.2442 & 1.3058 & 0.2154 & 0.7014 \\
\hline & & $(0.1129)$ & $(0.0264)$ & $(0.0435)$ & $(0.0941)$ & $(0.4679)$ & $(0.0778)$ & $(0.1329)$ \\
\hline & \multirow{2}{*}{600} & 0.1853 & 0.0665 & 0.0805 & 0.1852 & 1.1938 & 0.1736 & 0.7018 \\
\hline & & $(0.0691)$ & $(0.0211)$ & $(0.0285)$ & $(0.0799)$ & $(0.4903)$ & $(0.0587)$ & $(0.1083)$ \\
\hline \multirow{6}{*}{$5 \times 5$} & \multirow{2}{*}{200} & 0.6694 & 0.2576 & 0.2642 & 0.5813 & 1.7895 & 0.5770 & 0.9244 \\
\hline & & $(0.2213)$ & $(0.0647)$ & $(0.0658)$ & $(0.1815)$ & $(0.2317)$ & $(0.1535)$ & $(0.1684)$ \\
\hline & \multirow{2}{*}{400} & 0.4397 & 0.1483 & 0.1387 & 0.4008 & 1.7547 & 0.3821 & 0.8493 \\
\hline & & $(0.1317)$ & $(0.0393)$ & $(0.0366)$ & $(0.1197)$ & $(0.2561)$ & $(0.1084)$ & $(0.1302)$ \\
\hline & \multirow{2}{*}{600} & 0.3703 & 0.1221 & 0.1380 & 0.3166 & 1.6441 & 0.3013 & 0.8078 \\
\hline & & $(0.1041)$ & $(0.0305)$ & $(0.0366)$ & $(0.0770)$ & $(0.2984)$ & $(0.0872)$ & $(0.1233)$ \\
\hline \multirow{6}{*}{$7 \times 7$} & \multirow{2}{*}{200} & 0.9658 & 0.4182 & 0.4272 & 0.7788 & 1.9013 & 0.7529 & 1.1184 \\
\hline & & $(0.2799)$ & $(0.1185)$ & $(0.1000)$ & $(0.2764)$ & $(0.1407)$ & $(0.1802)$ & $(0.1708)$ \\
\hline & \multirow{2}{*}{400} & 0.6456 & 0.2185 & 0.2455 & 0.5012 & 1.8634 & 0.4998 & 0.9343 \\
\hline & & $(0.2046)$ & $(0.0484)$ & $(0.0448)$ & $(0.1236)$ & $(0.1761)$ & $(0.1143)$ & $(0.1102)$ \\
\hline & \multirow{2}{*}{600} & 0.5136 & 0.1664 & 0.1879 & 0.4176 & 1.8656 & 0.4073 & 0.8439 \\
\hline & & $(0.1157)$ & $(0.0338)$ & $(0.0410)$ & $(0.1162)$ & $(0.1708)$ & $(0.0902)$ & $(0.1004)$ \\
\hline
\end{tabular}

This example is a modification of Example 7.3 Model B of Yin and Li (2011). For this example, the dimensions of CDFS are $d_{0}=r_{0}=2$, which indicates the benchmark distances and the $5 \%$ critical values are the same as in Example 2. The basis matrices are $A=\left(e_{1}, e_{2}\right) \in \mathbb{R}^{p \times 2}$ and $B=\left(e_{1}, e_{2}\right) \in \mathbb{R}^{q \times 2}$ same as in Example 2 as well. The simulation results in Table 3 show that folded-MAVE ensemble and folded-SR ensemble are two comparable top performers. Based on benchmark distance, only folded-MAVE, folded-SR and folded-DR estimate the correct subspace. 
Table 2: Example 2. accuracy of estimates

\begin{tabular}{|c|c|c|c|c|c|c|c|c|}
\hline Size & $\mathrm{n}$ & f-OPG-E & f-MAVE-E & f-SR-E & folded-SIR & folded-SAVE & folded-DR & DF-PFC \\
\hline \multirow{6}{*}{$3 \times 3$} & \multirow{2}{*}{200} & 0.8795 & 0.5078 & 0.6942 & 1.5329 & 1.8388 & 1.1293 & 1.6025 \\
\hline & & $(0.4210)$ & $(0.3416)$ & $(0.4016)$ & $(0.5099)$ & $(0.4233)$ & $(0.5403)$ & $(0.4089)$ \\
\hline & \multirow{2}{*}{400} & 0.5744 & 0.2726 & 0.4210 & 1.3152 & 1.5606 & 0.6395 & 1.5617 \\
\hline & & $(0.2986)$ & $(0.1058)$ & $(0.2857)$ & $(0.5353)$ & $(0.5329)$ & $(0.3397)$ & $(0.3935)$ \\
\hline & \multirow{2}{*}{600} & 0.4370 & 0.2104 & 0.2865 & 1.2189 & 1.3844 & 0.4546 & 1.5321 \\
\hline & & $(0.1783)$ & $(0.0771)$ & $(0.2061)$ & $(0.5550)$ & $(0.5308)$ & $(0.2080)$ & $(0.4496)$ \\
\hline \multirow{6}{*}{$5 \times 5$} & \multirow{2}{*}{200} & 1.8910 & 1.2226 & 1.6211 & 2.0755 & 2.4045 & 2.0595 & 2.0991 \\
\hline & & $(0.3329)$ & $(0.4143)$ & $(0.3813)$ & $(0.3215)$ & $(0.2380)$ & $(0.3569)$ & $(0.2644)$ \\
\hline & \multirow{2}{*}{400} & 1.4537 & 0.6751 & 1.0256 & 1.8289 & 2.3139 & 1.4440 & 2.0111 \\
\hline & & $(0.2708)$ & $(0.2831)$ & $(0.3538)$ & $(0.4029)$ & $(0.2829)$ & $(0.4511)$ & $(0.2434)$ \\
\hline & \multirow{2}{*}{600} & 1.2988 & 0.4876 & 0.8864 & 1.6692 & 2.2178 & 1.0816 & 1.9152 \\
\hline & & $(0.2208)$ & $(0.1234)$ & $(0.3515)$ & $(0.3648)$ & $(0.3039)$ & $(0.3973)$ & $(0.2841)$ \\
\hline \multirow{6}{*}{$7 \times 7$} & \multirow{2}{*}{200} & 2.2103 & 1.8651 & 1.9234 & 2.2961 & 2.6258 & 2.2159 & 2.3368 \\
\hline & & $(0.1929)$ & $(0.3868)$ & $(0.1944)$ & $(0.2084)$ & $(0.1390)$ & $(0.2166)$ & $(0.1726)$ \\
\hline & \multirow{2}{*}{400} & 1.9403 & 1.2523 & 1.5485 & 2.1053 & 2.5348 & 2.0031 & 2.1895 \\
\hline & & $(0.2020)$ & $(0.4416)$ & $(0.3357)$ & $(0.2119)$ & $(0.2001)$ & $(0.3743)$ & $(0.2152)$ \\
\hline & \multirow{2}{*}{600} & 1.7327 & 0.8406 & 1.3545 & 1.9733 & 2.4768 & 1.7428 & 2.0955 \\
\hline & & $(0.2335)$ & $(0.4394)$ & $(0.3366)$ & $(0.2382)$ & $(0.1544)$ & $(0.4098)$ & $(0.1801)$ \\
\hline
\end{tabular}

In the above examples, as sample size increases, both folded-MAVE ensemble and foldedSR ensemble improve on estimating CDFS. Forward sufficient dimension reduction methods such as MAVE require larger sample size than the number of parameters. Large samples can provide accurate estimation. For a matrix/array-valued predictor, each element is a random variable. As the size of matrix or array increases, the number of random variables will increase dramatically. Folded MAVE and folded MAVE ensemble are developed based on MAVE, thus they also require large sample size. Obviously, comparing to the $5 \%$ critical value, our proposed methods can provide a good estimate of the CDFS for each of the three examples.

In the following Table 4, we report the percentages of correctly estimating the structural dimensions by the cross validation criterion based folded-MAVE ensemble estimator. The percentages of correctly estimating dimensions are consistently $100 \%$ for Example 1. For Example 2 , when $p=q=3$ and $p=q=5$, the correct percentages approach to $100 \%$ as sample size increases from 200 to 600 . As sample size increases, the estimated results by the cross validation criterion for $p=q=7$ improve as well. For Example 3, the results improve as sample size increases for each assignment of $p$ and $q$. 
Table 3: Example 3. accuracy of estimates

\begin{tabular}{|c|c|c|c|c|c|c|c|c|}
\hline Size & $\mathrm{n}$ & f-OPG-E & f-MAVE-E & f-SR-E & folded-SIR & folded-SAVE & folded-DR & DF-PFC \\
\hline \multirow{6}{*}{$3 \times 3$} & \multirow{2}{*}{200} & 1.2980 & 0.4676 & 0.4411 & 2.0894 & 1.4506 & 0.7682 & 1.9803 \\
\hline & & $(0.5235)$ & $(0.3432)$ & $(0.2181)$ & $(0.3053)$ & $(0.5785)$ & $(0.4155)$ & $(0.3326)$ \\
\hline & \multirow{2}{*}{400} & 0.7281 & 0.1640 & 0.2044 & 2.0729 & 1.1697 & 0.4426 & 2.0601 \\
\hline & & $(0.4186)$ & $(0.0649)$ & $(0.0819)$ & $(0.3009)$ & $(0.5195)$ & $(0.2424)$ & $(0.3133)$ \\
\hline & \multirow{2}{*}{600} & 0.3979 & 0.1264 & 0.1445 & 2.0764 & 0.89504 & 0.3094 & 2.0367 \\
\hline & & $(0.1863)$ & $(0.05769)$ & $(0.0582)$ & $(0.3773)$ & $(0.5745)$ & $(0.1325)$ & $(0.2909)$ \\
\hline \multirow{6}{*}{$5 \times 5$} & \multirow{2}{*}{200} & 2.4288 & 1.5986 & 1.5445 & 2.5836 & 2.3370 & 1.7116 & 2.4909 \\
\hline & & $(0.1951)$ & $(0.4574)$ & $(0.4176)$ & $(0.1403)$ & $(0.1829)$ & $(0.4415)$ & $(0.1842)$ \\
\hline & \multirow{2}{*}{400} & 2.4063 & 0.7791 & 0.7468 & 2.5934 & 2.1405 & 1.0427 & 2.4942 \\
\hline & & $(0.1702)$ & $(0.2767)$ & $(0.3329)$ & $(0.1430)$ & $(0.2958)$ & $(0.3424)$ & $(0.1695)$ \\
\hline & \multirow{2}{*}{600} & 2.3780 & 0.3059 & 0.3028 & 2.5862 & 1.8384 & 0.6865 & 2.5024 \\
\hline & & $(0.1846)$ & $(0.1269)$ & $(0.0680)$ & $(0.1312)$ & $(0.4369)$ & $(0.2089)$ & $(0.1641)$ \\
\hline \multirow{6}{*}{$7 \times 7$} & \multirow{2}{*}{200} & 2.5843 & 2.3894 & 2.3243 & 2.7082 & 2.5355 & 2.3853 & 2.6485 \\
\hline & & $(0.0988)$ & $(0.2180)$ & $(0.2693)$ & $(0.1040)$ & $(0.1254)$ & $(0.2871)$ & $(0.1023)$ \\
\hline & \multirow{2}{*}{400} & 2.5818 & 1.6047 & 1.6006 & 2.7058 & 2.3934 & 1.6116 & 2.6483 \\
\hline & & $(0.1180)$ & $(0.3003)$ & $(0.2973)$ & $(0.1101)$ & $(0.1043)$ & $(0.3743)$ & $(0.1230)$ \\
\hline & \multirow{2}{*}{600} & 2.5665 & 0.9993 & 0.9954 & 2.7134 & 2.3346 & 1.1680 & 2.6481 \\
\hline & & $(0.1323)$ & $(0.2889)$ & $(0.3596)$ & $(0.0897)$ & $(0.1576)$ & $(0.3271)$ & $(0.1140)$ \\
\hline
\end{tabular}

Table 4: Accuracy of cross validation on estimating CDFS dimensions

\begin{tabular}{ccccc}
\hline \multirow{2}{*}{ Example } & $\mathrm{n}$ & $\mathrm{p}=\mathrm{q}=3$ & $\mathrm{p}=\mathrm{q}=5$ & $\mathrm{p}=\mathrm{q}=7$ \\
& & & & \\
\hline \multirow{2}{*}{1} & 200 & $100 \%$ & $100 \%$ & $100 \%$ \\
& 400 & $100 \%$ & $100 \%$ & $100 \%$ \\
& 600 & $100 \%$ & $100 \%$ & $100 \%$ \\
\hline \multirow{2}{*}{2} & 200 & $62 \%$ & $47 \%$ & $27 \%$ \\
& 400 & $89 \%$ & $72 \%$ & $40 \%$ \\
& 600 & $96 \%$ & $94 \%$ & $74 \%$ \\
\hline \multirow{2}{*}{3} & 200 & $54 \%$ & $23 \%$ & $9 \%$ \\
& 400 & $75 \%$ & $64 \%$ & $34 \%$ \\
& 600 & $90 \%$ & $86 \%$ & $62 \%$ \\
\hline
\end{tabular}

\section{Application}

We now apply the folded-MAVE ensemble estimator to analyze a matrix-valued Standard \& Poor's 500 stock data set. We used the Web Spider technology to collect data from the html file of http://finance.sina.com.cn/stock/usstock/. The univariate response in the data set is the annually simple return rate for year 2014, which is assumed to be continuous and independent between each stock. If $P_{i 0}$ and $P_{i 1}$ denote the $i$ th stock's price at the beginning and end of the 
year 2014 , then the responses $Y_{i}=\left(P_{i 1}-P_{i 0}\right) / P_{i 0}, i=1, \ldots, n$, where $n$ is the sample size. The predictor variable is a $5 \times 4$ matrix which is formed by five indexes in four quarterly financial reports in year 2014. The five indexes are profit margin (PM), return on sales (ROS), debt ratio (DebtR), return on total assets (ROTA) and return on equity (ROE). There are 88 stocks that miss information on one or two of the five indexes leaving $n=412$ observations with full record. The primary interest is investigating the association between annually simple return rate and the combination of the five indexes and the time points.

We adopt the prediction approach proposed by Ding and Cook (2014, Section 5) to consider the prediction of $Y$ from $\operatorname{vec}(X)$. That approach regresses $Y$ on $\operatorname{vec}(X)$ in two steps. In the first step, by applying dimension folding methods, one can obtain the estimate of the CDFS. After dimension folding, one has a new reduced predictor $\hat{A}^{\top} X \hat{B}$ with smaller row and column dimensions compared to the original predictor $X$. The second step is fitting a generalized additive model (GAM, Hastie and Tibshirani, 1990; Wood, 2000, 2006, 2008) to estimate the mean function $E\left[Y \mid \operatorname{vec}\left(\hat{A}^{\top} X \hat{B}\right)\right]$. The prediction can be carried out by fitting a generalized additive model of $Y_{i}^{*}$ on the reduced predictor $(\hat{B} \otimes \hat{A})^{\top} \operatorname{vec}\left(X_{i}^{*}\right)$ for observations $\left(X_{i}^{*}, Y_{i}^{*}\right), i=1, \ldots, n_{p}$. The prediction error is calculated as

$$
P E=\sum_{i=1}^{n_{p}}\left[Y_{i}^{*}-\hat{E}\left(Y \mid \operatorname{vec}\left(\hat{A}^{\top} X_{i}^{*} \hat{B}\right)\right]^{2} / n_{p}\right.
$$

We apply the cross validation criterion in Section 3.4 to estimate the structural dimensions of CDFS and $\hat{d_{0}}=2$ and $\hat{r_{0}}=3$. We run a 10-fold cross validation method to evaluate the prediction errors. We repeat the 10-fold cross validation method for 1000 times and use the average of the prediction error, $\sum_{i=1}^{1000} P E_{i} / 1000$, to assess the performances of folded-MAVE ensemble, foldedSIR, folded-SAVE, folded-DR and isotropic DF-PFC. The results are summarized in Table 5. It can be seen that folded-MAVE ensemble estimator provides a smaller prediction error than all other methods. From the estimated basis matrices (not reported) of CDFS, we notice that ROTA and ROE play more important roles than the other three variables. The basis matrices reveal that the first three report have stronger connection with the annual return but the fourth report not. Usually, the financial report is released one or two month later, which means that the fourth quarter report will not released until January or February or even later in year 2015. We think releases of the reports will affect stock price.

Table 5: 10-fold cross validation prediction errors $(\times 10)$

\begin{tabular}{llllr}
\hline f-MAVE-E & folded-SIR & folded-SAVE & folded-DR & DF-PFC \\
\hline 0.6840 & 1.2193 & 2.5176 & 1.1884 & 0.8681 \\
\hline
\end{tabular}




\section{Generalization to array-valued predictors}

We now briefly discuss the extension of ensemble idea of sufficient dimension folding to a array-valued predictor. Let $\mathbf{X}=\left\{X_{j_{1} j_{2} \ldots j_{k}}: j_{1}=1, \ldots, p_{1}, \ldots, j_{k}=1, \ldots, p_{k}\right\}$ be a $k$-way random array of dimension $p_{1} \times \cdots \times p_{k}$. The CDFS and CMDFS of $Y$ conditional on the arrayvalued $\mathbf{X}$ are denoted by $\mathcal{S}_{Y \mid \mathbf{X} \circ^{k}}(\mathrm{LKA}, 2010)$ and $\mathcal{S}_{E\left(Y \mid \mathbf{X}_{\circ^{k}}\right)}$ (Xue and Yin, 2014), respectively. The ensemble idea is applying a family of functions $f(\cdot)$ to $Y$ and taking the unison of several $\mathcal{S}_{E\left[f(Y) \mid \mathbf{X}_{\left.\circ^{k}\right]}\right.}$ to approximate $\mathcal{S}_{Y \mid \mathbf{X}_{\circ} k}$. We have a definition parallel to Definition 1 as follows.

Definition 2. If $\mathfrak{F}$ satisfies

$$
\operatorname{span}\left\{\mathcal{S}_{E\left[f(Y) \mid \mathbf{X}_{\left.\circ^{k}\right]}\right]}: f \in \mathfrak{F}\right\}=\mathcal{S}_{Y \mid \mathbf{X}_{\circ^{k}}},
$$

the family $\mathfrak{F}$ is said to be characterizing the CDFS on the $k$-way array-valued $\mathbf{X}$.

Following LKA (2010) and Yin and Li (2011), we can easily prove that if $\mathfrak{F}$ is a subset of $L_{2}\left(F_{Y}\right)$ that is dense in $\mathfrak{B}, \mathfrak{F}$ can characterize the CDFS for the $k$-way array-valued $\mathbf{X}$, and $\mathcal{S}_{Y \mid \mathbf{X} \circ^{k}}$ can be characterized by a finite number of functions of the response variable in a characterizing family. Similar ensemble approaches on estimating $\mathcal{S}_{Y \mid \mathbf{X} \circ^{k}}$ can be constructed analogously and we omit the details of algorithms for brevity.

\section{Discussion}

In this paper, we establish sufficient dimension folding methods based on the ensemble idea to estimate CDFS. Inspired by the fused estimators (Cook and Zhang, 2014; Wu and Yin, 2015), we construct folded-SR ensemble method which adopts a series of numbers of slices to estimate CDFS. Simulations show that folded-MAVE ensemble and folded-SR ensemble overtake folded methods proposed by LKA (2010) and DF-PFC with isotropic errors in Ding and Cook (2014). Ensemble idea by repeatedly using the data will certainly enhance the accuracy of estimates, as demonstrated in our simulations. We analyze a Standard \& Poor's 500 stock data set to show the efficacy of folded-MAVE ensemble. We believe that such an approach can be useful in many areas with matrix-/array-valued predictors.

\section{Acknowledgement}

The authors would like to thank the Editor, the Associate Editor and the three referees for their constructive and insightful comments and suggestions that greatly improved the paper. Xue's research was partly supported by the NSF-China grant (No. 11401095), National Bureau of Statistics of China grant (2014LY127), the Fundamental Research Funds for the Central Universities (16YQ09), Scientific Research Project Achievement of networking and collaboration 
center for China's multinational business (201504YY006A) at UIBE, and Zhejiang province social science grant (16NDJC154YB). Yin's research was partly supported by the NSF grant (No. 1205546).

\section{Appendix}

Appendix A: Proof of Theorem 1.

This proof follows the proof for Theorem 2.1 in Yin and Li (2011). We know that $\mathfrak{F}$ is a subset of $L_{1}\left(F_{Y}\right)$ and $L_{2}\left(F_{Y}\right)$ as well. By Lemma 2.1 in Yin and Li (2011), it suffices to show that Equation (1) satisfied for all $f \in \mathfrak{F}$ implies Equation (2).

Let $\mathcal{B}$ be a Borel set in $\Omega_{Y}$, and let $\mathcal{S}_{L}$ and $\mathcal{S}_{R}$ be the subspaces such that Equation (1) holds for all $f \in \mathfrak{F}$. Since $\mathfrak{F}$ is dense in $\mathfrak{B}$ there exists a sequence $\left\{f_{k}\right\} \subseteq \mathfrak{F}$ such that $\lim _{k \rightarrow \infty} E\left[I_{\mathcal{B}}(Y)-\right.$ $\left.f_{k}(Y)\right]^{2}=0$. For any $g \in L_{2}\left(F_{X}\right)$, there exists

$$
\begin{aligned}
E\left\{g(X)\left[I_{\mathcal{B}}(Y)-E\left(I_{\mathcal{B}}(Y) \mid P_{\mathcal{S}_{L}} X P_{\mathcal{S}_{R}}\right)\right]\right\}= & E\left\{g(X)\left[I_{\mathcal{B}}(Y)-E\left(f_{k}(Y) \mid P_{\mathcal{S}_{L}} X P_{\mathcal{S}_{R}}\right)\right]\right\} \\
& +E\left\{g(X) E\left[f_{k}(Y)-I_{\mathcal{B}}(Y) \mid P_{\mathcal{S}_{L}} X P_{\mathcal{S}_{R}}\right]\right\} .
\end{aligned}
$$

Because $f_{k} \in \mathfrak{F}$ we have $E\left[f_{k}(Y) \mid P_{\mathcal{S}_{L}} X P_{\mathcal{S}_{R}}\right]=E\left[f_{k}(Y) \mid X\right]$. By the same argument in Yin and Li (2011), we can show that the first term on the right-hand side of Equation (8) is no more than $E\left[g^{2}(X)\right] E\left\{\left[I_{\mathcal{B}}(Y)-f_{k}(Y)\right]^{2}\right\} \rightarrow 0$, as $k \rightarrow \infty$. The square of the second term on the right-hand side of Equation (8) is no more than

$$
E\left[g^{2}(X)\right] E\left\{E^{2}\left[f_{k}(Y)-I_{\mathcal{B}}(Y) \mid P_{\mathcal{S}_{L}} X P_{\mathcal{S}_{R}}\right]\right\} \leq E\left[g^{2}(X)\right] E\left\{E\left[f_{k}(Y)-I_{\mathcal{B}}(Y)\right]^{2}\right\} \rightarrow 0,
$$

as $k \rightarrow \infty$.

Because the left-hand side of Equation (8) does not depend on $k$, and the right-hand side converges to 0 as $k \rightarrow \infty$, we have $E\left\{g(X)\left[I_{\mathcal{B}}(Y)-E\left(I_{\mathcal{B}}(Y) \mid P_{\mathcal{S}_{L}} X P_{\mathcal{S}_{R}}\right)\right]\right\}=0$ for all $g \in L_{2}\left(F_{X}\right)$, which implies $E\left[I_{\mathcal{B}}(Y) \mid P_{\mathcal{S}_{L}} X P_{\mathcal{S}_{R}}\right]=E\left[I_{\mathcal{B}}(Y) \mid X\right]$ almost surely. Since $\mathcal{B}$ is an arbitrary Borel set in $\Omega_{X}$, we have $Y \Perp X \mid P_{\mathcal{S}_{L}} X P_{\mathcal{S}_{R}}$. The proof is complete.

Appendix B: Proof of Theorem 2.

For $u=1, \ldots, d_{0}$ and $v=1, \ldots, r_{0}$, let $\Lambda_{u}$ and $\Lambda_{v}$ be subsets of $\left\{t \in \Omega_{T}\right\}$ such that $\beta_{u} \notin \operatorname{span}\left\{\mathcal{S}_{E\left[f_{t}(Y) \mid \circ X\right]}: t \in \Lambda_{u}\right\}$ and $\beta_{v} \notin \operatorname{span}\left\{\mathcal{S}_{E\left[f_{t}(Y) \mid X \circ\right]}: t \in \Lambda_{v}\right\}$, respectively. If $P\left(T \in \Lambda_{u}\right)=1$ for some $u$ or $P\left(T \in \Lambda_{v}\right)=1$ for some $v$, then $\mathfrak{F}$ does not characterize $\mathcal{S}_{Y \mid \circ X \circ}$, which is a contradiction. Hence $P\left(T \in \Lambda_{u}\right)<1$ for $u=1, \ldots, d_{0}$ and $P\left(T \in \Lambda_{v}\right)<$ 1 for $v=1, \ldots, r_{0}$. Let $A\left(T_{1: m}\right)=A\left(T_{1}, \ldots, T_{m}\right)$ and $B\left(T_{1: m}\right)=B\left(T_{1}, \ldots, T_{m}\right)$. And let $\delta_{1}\left(T_{1}, \ldots, T_{m}\right)=\left\|A\left(T_{1: m}\right) A^{\top}\left(T_{1: m}\right)-A A^{\top}\right\|, \delta_{2}\left(T_{1}, \ldots, T_{m}\right)=\left\|B\left(T_{1: m}\right) B^{\top}\left(T_{1: m}\right)-B B^{\top}\right\|$ and $\delta\left(T_{1}, \ldots, T_{m}\right)=\left\|\left[B\left(T_{1: m}\right) \otimes A\left(T_{1: m}\right)\right]\left[B\left(T_{1: m}\right) \otimes A\left(T_{1: m}\right)\right]^{\top}-(B \otimes A)(B \otimes A)^{\top}\right\|$. 
We have $\delta\left(T_{1}, \ldots, T_{m}\right)=0$ if and only if $\delta_{1}\left(T_{1}, \ldots, T_{m}\right)=0$ and $\delta_{2}\left(T_{1}, \ldots, T_{m}\right)=0$. That is for some $u=1, \ldots, d_{0}, T_{1}, \ldots, T_{m}$ all belong to $\Lambda_{u}$ and for some $v=1, \ldots, r_{0}, T_{1}, \ldots, T_{m}$ all belong to $\Lambda_{v}$, which are the event $\cup_{u=1}^{d_{0}} \cap_{k=1}^{m}\left\{T_{k} \in \Lambda_{u}\right\}$ and $\cup_{v=1}^{r_{0}} \cap_{k=1}^{m}\left\{T_{k} \in \Lambda_{v}\right\}$. The probabilities of the two events are less or equal to $\sum_{u=1}^{d_{0}}\left[P\left(T \in \Lambda_{u}\right)\right]^{m}$ and $\sum_{v=1}^{r_{0}}\left[P\left(T \in \Lambda_{v}\right)\right]^{m}$, respectively.

Since

$$
\begin{gathered}
\sum_{m=1}^{\infty} \sum_{u=1}^{d_{0}}\left[P\left(T \in \Lambda_{u}\right)\right]^{m}=\sum_{u=1}^{d_{0}} \frac{P\left(T \in \Lambda_{u}\right)}{1-P\left(T \in \Lambda_{u}\right)}<\infty, \text { and } \\
\sum_{m=1}^{\infty} \sum_{v=1}^{r_{0}}\left[P\left(T \in \Lambda_{v}\right)\right]^{m}=\sum_{v=1}^{r_{0}} \frac{P\left(T \in \Lambda_{v}\right)}{1-P\left(T \in \Lambda_{v}\right)}<\infty,
\end{gathered}
$$

by the first Borel-Cantelli lemma, we have with probability 1,

$$
\lim _{m \rightarrow \infty} \delta_{1}\left(T_{1}, \ldots, T_{m}\right)=0, \text { and } \lim _{m \rightarrow \infty} \delta_{2}\left(T_{1}, \ldots, T_{m}\right)=0
$$

Since $\operatorname{span}\left[A\left(T_{1}, \ldots, T_{m}\right)\right] \subseteq \operatorname{span}(A)$ and $\operatorname{span}\left[B\left(T_{1}, \ldots, T_{m}\right)\right] \subseteq \operatorname{span}(B)$, by Lemma 2.2 in Yin and $\operatorname{Li}(2011)$, Equation (9) is true if and only if $\delta_{1}\left(T_{1}, \ldots, T_{m}\right)=0$ and $\delta_{2}\left(T_{1}, \ldots, T_{m}\right)=0$ for sufficiently large $m$. Therefore, with probability 1 , there exists an $m_{0}(\omega)$ such that for $m>m_{0}(\omega), \operatorname{span}\left[A\left(T_{1}, \ldots, T_{m}\right)\right]=\operatorname{span}(A)$ and $\operatorname{span}\left[B\left(T_{1}, \ldots, T_{m}\right)\right]=\operatorname{span}(B)$. This completes the proof.

\section{Appendix C: Proof of Theorem 5.}

By the proof of Theorem 2, we know that $\operatorname{span}\left\{A\left(T_{1}, \ldots, T_{m}\right)\right\} \subseteq \operatorname{span}(A), \operatorname{span}\left\{B\left(T_{1}, \ldots, T_{m}\right)\right\} \subseteq$ $\operatorname{span}(B)$, and $\delta\left(T_{1}, \ldots, T_{m}\right)$ becomes 0 for sufficiently large $m$, which means that $\left[d\left(T_{1: m}\right), r\left(T_{1: m}\right)\right]$ is equal to $(d, r)$ for sufficiently large $m$. Consequently, $P\left(\liminf _{m \rightarrow \infty}\left\{\left[d\left(T_{1: m}\right), r\left(T_{1: m}\right)\right]=\left(d_{0}, r_{0}\right)\right\}\right)=$ 1. By Fatou's lemma,

$$
\begin{aligned}
\liminf _{m \rightarrow \infty} P( & {\left.\left[d\left(T_{1: m}\right), r\left(T_{1: m}\right)\right]=\left(d_{0}, r_{0}\right)\right) } \\
& \geq P\left(\liminf _{m \rightarrow \infty}\left\{\left[d\left(T_{1: m}\right), r\left(T_{1: m}\right)\right]=\left(d_{0}, r_{0}\right)\right\}\right)=1
\end{aligned}
$$

Thus $\lim _{m \rightarrow \infty} P\left(\left\{\left[d\left(T_{1: m}\right), r\left(T_{1: m}\right)\right]=\left(d_{0}, r_{0}\right)\right\}\right)=1$. Since $d\left(T_{1: m}\right)$ and $r\left(T_{1: m}\right)$ do not depend on $n$, we have

$$
\lim _{m \rightarrow \infty} \lim _{n \rightarrow \infty} P\left(\left[d\left(T_{1: m}\right), r\left(T_{1: m}\right)\right]=\left(d_{0}, r_{0}\right)\right)=1 .
$$

Since $T_{1}, \ldots, T_{m}$ are independent of $\left\{\left(x_{i}, y_{i}\right), i=1, \ldots, n\right\}$, and

$$
\lim _{n \rightarrow \infty} P\left(\left[\hat{d}\left(T_{1: m}\right), \hat{r}\left(T_{1: m}\right)\right]=\left[d\left(T_{1: m}\right), r\left(T_{1: m}\right)\right] \mid T_{1}=t_{1}, \ldots, T_{m}=t_{m}\right)=1,
$$


by the dominated convergence theorem we have

$$
\lim _{n \rightarrow \infty} P\left(\left[\hat{d}\left(T_{1: m}\right), \hat{r}\left(T_{1: m}\right)\right]=\left[d\left(T_{1: m}\right), r\left(T_{1: m}\right)\right]\right)=1,
$$

which implies

$$
\lim _{m \rightarrow \infty} \lim _{n \rightarrow \infty} P\left(\left[\hat{d}\left(T_{1: m}\right), \hat{r}\left(T_{1: m}\right)\right]=\left[d\left(T_{1: m}\right), r\left(T_{1: m}\right)\right]\right)=1 .
$$

And further we have

$$
\lim _{m \rightarrow \infty} \lim _{n \rightarrow \infty} P\left(\left[\hat{d}\left(T_{1: m}\right), \hat{r}\left(T_{1: m}\right)\right]=\left(d_{0}, r_{0}\right)\right)=1 .
$$

The proof is complete.

\section{References}

Cook, R. D., 1994. On the interpretation of regression plots. Journal of the American Statistical Association, 89, 177-190.

Cook, R. D., 1996. Graphics for regressions with a binary response. Journal of the American Statistical Association, 91, 983-992.

Cook, R. D., 1998. Principal Hessian directions revisited (with discussion). Journal of the American Statistical Association, 93, 84-C100.

Cook, R. D. and Weisberg, S., 1991. Discussion of Sliced inverse regression for dimension reduction, by K. C. Li. Journal of the American Statistical Association, 86, 328-332.

Cook, R. D. and Zhang, X., 2014. Fused estimators of the central subspace in sufficient dimension reduction. Journal of the American Statistical Association, 19, 815-827.

Ding, S. and Cook, R. D., 2014. Dimension folding PCA and PFC for matrix-valued predictors. Statistica Sinica, 24, 463-492.

Fan, J., and Gijbels, I., 1996. Local Polynomial Modelling and Its Applications. London: Chapman \& Hall.

Fan, J., Yao, Q. and Cai, Z., 2003. Adaptive varying-coefficient linear models. Journal of the Royal Statistical Society Series B, 65, 57-80.

Hastie, T. J. and Tibshirani, R. J., 1990. Generalized Additive Models. 1st Ed. London: Chapman \& Hall.

Li, B., Kim, M. and Altman, N., 2010. On dimension folding of matrix- or array-valued statistical objects. The Annals of Statistics, 38, 1094-1121. 
Li, B, Wen, S. and Zhu, L., 2008. On a projective resampling method for dimension reduction with multivariate responses. Journal of the American Statistical Association, 103, 11771186.

Li, B., Zha, H. and Chiaromonte, F., 2005. Contour Regression: a general approach to dimension reduction. The Annals of Statistics, 33, 1580-1616.

Li, K.-C., 1991. Sliced inverse regression for dimension reduction. Journal of the American Statistical Association, 86, 316-342.

Pfeiffer, M. R., Forzani, L. and Bura, E., 2011. Sufficient dimension reduction for longitudinally measured predictors. Statistics in Medicine, 22, 2414-2427.

Scott, D. W., 1992. Multivariate Density Estimation: Theory, Practice, and Visualization. 1st Ed. New York: Wiley.

Wang, H. and Xia, Y., 2008. Sliced regression for dimension reduction. Journal of the American Statistical Association, 103, 811-821.

Wood, S. N., 2000. Modelling and smoothing parameter estimation with multiple quadratic penalties. Journal of the Royal Statistical Society Series B, 62 (2), 413-428.

Wood, S. N., 2006. Generalized Additive Models: An Introduction with R. 1st Ed. London: Chapman \& Hall.

Wood, S. N., 2008. Fast stable direct fitting and smoothness selection for generalized additive models. Journal of the Royal Statistical Society Series B, 70 (3), 495-518.

Wu, W. and Yin, X., 2015. Stable estimation in dimension reduction. Journal of Computational and Graphical Statistics, 24 (1), 104-120.

Xia, Y., 2007. A constructive approach to the estimation of dimension reduction directions. The Annals of Statistics, 35, 2654-2690.

Xia, Y., Tong, H., Li, W. and Zhu, L., 2002. An adaptive estimation of dimension reduction. Journal of Royal Statistical Society Series B, 64, 363-410.

Xue, Y. and Yin, X., 2014. Sufficient dimension folding for regression mean function. Journal of Computational and Graphical Statistics, 23, 1028-1043.

Xue, Y. and Yin, X., 2015. Sufficient dimension folding for a functional of conditional distribution of matrix- or array-valued objects. Journal of Nonparametric Statistics, 27, 253-269. 
Yin. X. and Li, B., 2011. Sufficient dimension reduction based on an ensemble of minimum average variance estimators. The Annals of Statistics, 39, 3392-3416.

Zhu, Y. and Zeng, P., 2006. Fourier methods for estimating the central subspace and the central mean subspace in regression. Journal of the American Statistical Association, 101, $1638-1651$. 